\title{
CONSUMPTION OF TOMATOES REDUCES THE CARDIOVASCULAR DISEASE
}

\author{
Professor Asim K. Duttaroy \\ Faculty of Medicine, University of Oslo, POB 1046 Blindern, N-0316 Oslo, Norway \\ Email: a.k.duttarov@medisin.uio.no
}

\begin{abstract}
:
Emerging epidemiological and intervention data support the connection between increased tomato consumption and reduced risk for cardiovascular disease. Tomato contains several antioxidants including lycopene, polyphenols, trace elements, and vitamins those are known to inhibit atherosclerotic processes. Recent studies have demonstrated that tomato also contains several potent anti-platelet compounds. The ingestion of these tomato components significantly affects ex vivo platelet function when supplemented in human volunteers. Platelet aggregation is a central mechanism in the pathogenesis of acute coronary syndromes, including myocardial infarction and unstable angina. Since tomato can reduce both platelet hyperactivity and atherosclerosis process thus makes it an ideal food for the cardiovascular system. Additionally tomato has the lowest content of uric acid than any fruits and vegetables. Tomato extract, commercially known as Fruitflow, is an absolutely unique dietary solution for cardiovascular support that works immediately after ingestion. Fruitflow works by helping to keep blood platelets smooth, thereby promoting healthy circulation and pressure within the cardiovascular system.
\end{abstract}

\section{INTRODUCTION}

During the last 50 years, the fruit of the cultivated tomato (Lycopersicon esculentum) has become a popular and highly consumed food[1]. Tomato contains several components that are beneficial to overall health, including vitamin E, trace elements, flavonoids, phytosterols, and several watersoluble vitamins[2-4]. Tomato is also a rich source of folate, vitamin C, and potassium[3,5]. Moreover, the antioxidant activity of lycopene as well as several other carotenoids and their abundance in tomato, makes it a rich source of antioxidants[2-4]. In addition, vitamin A precursors such as beta- and gamma-carotene are present in modest levels in tomatoes. 
Table 1: Major Constituents of Tomatoes

\begin{tabular}{|c|c|c|c|c|c|}
\hline Nutrients & $\begin{array}{l}\text { Raw } \\
\text { tomatoes }\end{array}$ & Catsup & Tomato juice & Tomato sauce & Tomato soup \\
\hline Potassium, mg & 237 & 382 & 229 & 331 & 181 \\
\hline$\alpha$-tocopherol, mg & 0.54 & 1.46 & 0.32 & 2.08 & 0.50 \\
\hline Viatmin A, IU & 833 & 933 & 450 & 348 & 193 \\
\hline Vitamin C, mg & 12.7 & 15.1 & 18.3 & 7.0 & 27.3 \\
\hline Folate, ? & 15 & 15 & 20 & 9 & 7 \\
\hline$\beta$ carotene, ?g & 449 & 560 & 270 & 290 & 75 \\
\hline$\alpha$ carotene, 回g & 101 & 0 & 0 & 0 & 0 \\
\hline Lycopene, ?]g & 2573 & 17007 & 9037 & 15152 & 5084 \\
\hline Lutein+zeaxanthin, 国g & 123 & 0 & 60 & 0 & 1 \\
\hline Phytoene, [?g & 1860 & 3390 & 1900 & 2950 & 1720 \\
\hline Phytofluene, 国g & 820 & 1540 & 830 & 1270 & 720 \\
\hline
\end{tabular}

\section{\# Adapted from [1]}

Table-1 shows the major constituents of tomatoes. Many of these nutrients may act individually, or in concert, to protect cells via several mechanisms including inhibition of free radical mediated oxidative damages, the most widely accepted theory for the genesis of atherosclerosis. Other cardioprotective functions provided by the nutrients in tomatoes may include the reduction of lowdensity lipoprotein (LDL) cholesterol, homocysteine, platelet activity, and blood pressure. Because tomatoes include several nutrients associated with theoretical or proven effects and are widely consumed year round, they may be considered a valuable component of a cardioprotective diet. The antioxidant and free radical-scavenging properties of polyphenol compounds have possible protective roles in reducing risk of cardiovascular diseases in humans. In fact, individuals in the Mediterranean area have a lower risk of several important chronic diseases, including cardiovascular disease (CVD) and a number of types of cancer (breast, colon, and prostate cancer) when compared 
with their North American and other European counterparts[2,6,7]. These differences may be associated with nutritional traditions. Consumption of tomatoes appears to be one such tradition that may account for the lower risk associated with diseases in people from this geographical area $[2,6,7]$. In addition to vitamins and antioxidants, water soluble anti-platelet compounds present in tomatoes are capable of inhibiting platelet aggregation both in vitro and in vivo [8]. Therefore the presence of both antioxidants and newly identified anti-thrombotic compounds makes tomato a beneficial fruit in preventing CVD. There are several excellent reviews available on overall health benefits of tomatoes, including on cardiovascular health [1,6,9-11]; however, this review will focus mainly on the beneficial effects of tomato on the cardiovascular system with special reference to platelets, one of the important limb of the cardiovascular system.

\section{EPIDEMIOLOGY}

Recent epidemiologic studies have focused on tomato and tomato products associated their intake with a reduced risk of degenerative diseases $[9,12,13]$. There is epidemiological evidence that consumers of tomatoes have a lower risk of many types of chronic diseases, including CVD and different forms of cancer[6,14,15]. The Health Professionals Follow Up Study first showed that high intake of tomato based products was linked to lower prostate cancer risk[9,12,13][16]. There is evidence in support of the role of lycopene in the prevention of CVD stems primarily from epidemiological observations on normal and at-risk populations[17-21]. The Mediterraneans have a lower incidence of chronic diseases in particular, CVD[10]. In recent epidemiological studies, tissue and serum levels of lycopene were inversely related to the risk of CVD.

The strongest population-based evidence for the beneficial effects of lycopene comes from a multicentre case-control study (EURAMIC). Subjects (662 cases and 717 controls) from 10 European countries were recruited to maximize variability in exposure within the study[22-24]. After adjusting for age, body mass index, socioeconomic status, smoking, hypertension, and maternal and paternal history of the disease, only lycopene, and not ß-carotene levels was found to be protective against CVD. The protective potential of lycopene was maximal among individuals with the highest polyunsaturated fat stores[23]. These results are consistent with a protective effect of adipose tissue levels of lycopene on myocardial infarction risk. In a cross-sectional study comparing Lithuanian and Swedish populations showing diverging mortality rates for CVD, lower blood lycopene levels were found to be associated with increased risk and mortality from CVD[25]. In an Austrian stroke

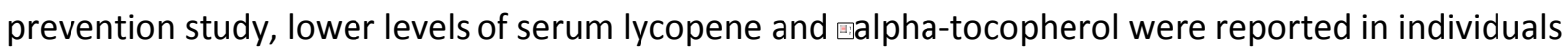
from an elderly population at high risk for microangiopathy-related cerebral damage[26]. 


\section{BENEFICIAL EFFECTS OF TOMATOES ON THE CARDIOVASCULAR SYSTEM}

\section{a. Effects of tomatoes on atherosclerosis}

Antioxidants are believed to slow the progression of atherosclerosis because of their ability to inhibit damaging oxidative processes. Lycopene has been suggested to have several mechanisms of action including inhibition of LDL oxidation[27]. Lycopene, because of its high number of conjugated dienes, is the most potent singlet oxygen quencher among the natural carotenoids[28]. Although evidence for a relationship between in vitro LDL oxidation and risk of CVD is not fully established, LDL oxidation is now recognized as representing an important early event in the development of atherosclerosis. Other possible mechanisms include enhanced LDL degradation, LDL particle size and composition, plaque rupture, and altered endothelial functions. However, there is convincing evidence to indicate that reactive oxygen species (ROS) generated both endogenously and also in response to diet and lifestyle factors may play a significant role in the aetiology of atherosclerosis and CVD. Central to this oxidative hypothesis is oxidation of LDL as the primary initial step leading to its uptake by macrophages inside the arterial wall and, formation of foam cells and atherosclerotic plaque. Although genetic factors and age are important in determining risk, several other factors, including hypertension, hypercholesterolemia, insulin resistance, smoking and diet are also major risk factors associated with CVD[29-31]. Oxidative stress induced by ROS is also considered to play an important role in the aetiology of several chronic diseases, including CVD[32-34]. Oxidised LDL (oxLDL) is thought to play a key role in the pathogenesis of atherosclerosis[32-34]. As a result of oxidative modifications of the native LDL molecule, several biologically active molecules can be formed, including protein adduct products with breakdown products of oxidized fatty acids that facilitate recognition of modified LDL by macrophage scavenger receptors. In addition to influencing the formation of foam cells and plaque in arterial walls, components of oxLDL can also influence other events that are related to increased risk of CVD. Oxidised components of LDL can promote migration and proliferation of smooth muscle cells, formation of foam cells and fatty streaks in the arterial intima and, cause eventual rupture of arterial plaques. Therefore, inhibition of LDL oxidation may play an important role in preventing CVD. Consumption of lycopene reduced serum lipid peroxidation and LDL cholesterol oxidation as the serum lycopene levels increased[2]. However, despite an inverse association found for high intakes of tomato-based products, dietary lycopene was not strongly associated with the risk of CVD. This indicates that other unidentified compounds in tomatoes may have cardioprotective effects. 
Increased risk of CVD has been associated with elevated triglycerides, total cholesterol, and LDL-C and with decreased HDL-C. Flavonoids are very efficient scavengers of peroxyl radicals, and the action could be related to their capacity to reduce and chelate ferric iron, which catalyzes lipid peroxidation. Tomatoes contain a variety of polyphenols, such as quercetin, kaempferol, and naringenin, which are potent antioxidants. Clinical evaluation showed that plasma antioxidation and phenolic contents were increased after administration of fresh tomato and tomato juice [35]. TG levels were decreased after administration of fresh tomato and tomato juice. HDL-C was found to increase whereas LDL-C was found to decrease after the consumption of fresh tomato and tomato juice. This clearly revealed that tomato phenolics probably played an important role in health promotion. High intake of tomato juice prevents LDL oxidation and thiobarbituric reactive species formation in healthy men. In general, circulating and adipose tissue levels of lycopene seem to be better indicators of disease prevention than dietary intake data. Lycopene has been shown to be absorbed better from processed tomato products than from fresh tomatoes.

\section{b. Anti-thrombotic factors in tomatoes}

Platelet aggregation is fundamental to a wide range of physiological and pathological processes, including the induction of thrombosis and arteriosclerosis[36-38]. Normal haemostasis is initiated when platelets are exposed to the sub-endothelial matrix, where they adhere to collagen via specific cell-surface receptors[39-42]. This adhesion step is followed by platelet activation that is accompanied by synthesis and release of pro-aggregatory molecules such as thromboxane (Tx) $A_{2}$ and ADP, which amplify platelet responses to collagen and recruit additional platelets to the site of injury[43-46]. The concerted action of collagen, ADP, and $\mathrm{TxA}_{2}$ activates specific signalling pathways, generating a number of second messengers and leads to functional expression of a GPIIb-GPIIla complex of the fibrinogen receptor on the platelets[43-46].

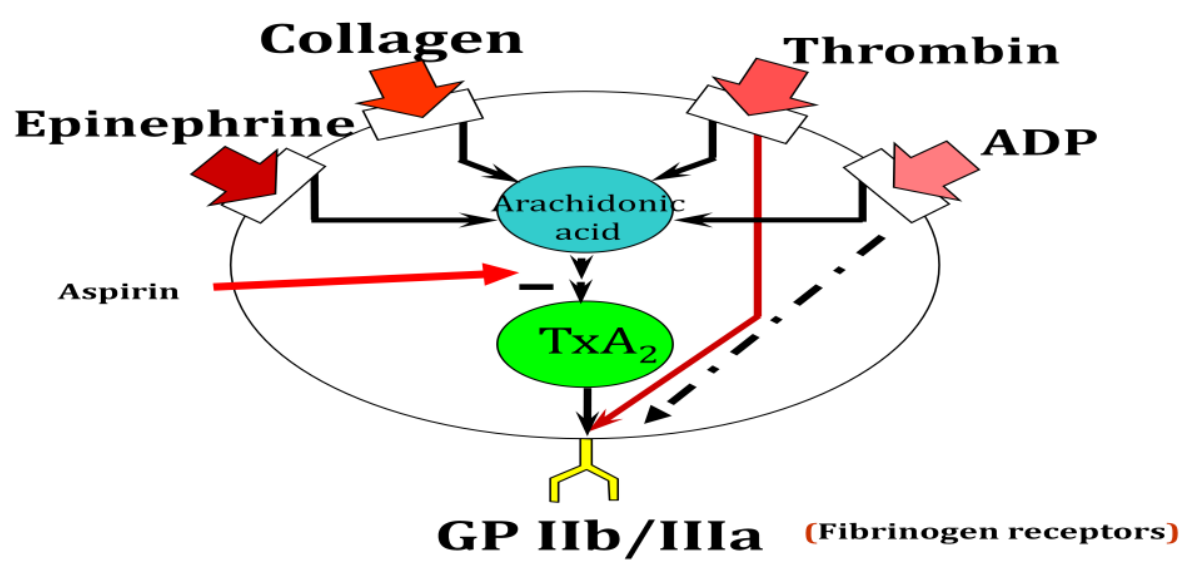


Fig. 1 shows the activation of platelets by different aggregating agents. Pro-haemostatic mechanisms appear to be counterbalanced and regulated by a number of physiologic anti-haemostatic molecules that work in a concerted and redundant manner, resulting in the release of prostacyclin $\left(\mathrm{PGI}_{2}\right)$, nitric oxide, and endothelium-dependent hyperpolarizing factor by the endothelium as well as ADP hydrolyzing activity associated with endothelial cell membrane apyrase (CD39)[46-49]. Aggregation of platelets by agonists is mediated, in part, through the intracellular formation of prostaglandin (PG) $\mathrm{G}_{2}, \mathrm{PGH}_{2}$ and $\mathrm{TxA}_{2}$ from arachidonic acid, 20:4n-6[46-49]. In contrast, $\mathrm{PGI}_{2}$, an arachidonic acid metabolite of endothelial cells, is the most potent natural inhibitor of platelet aggregation. Prostaglandin-induced inhibition of platelet aggregation is mediated through an increase in cAMP synthesis due to activation of adenylate cyclase. Activation of adenylate cyclase is initiated by binding of $\mathrm{PGI}_{2}$ or $\mathrm{PGE}_{1}$ to specific platelet surface receptors. Alternatively, cAMP levels in platelets can also be increased by inhibiting cAMP phosphodiesterase activity[46-49].

Hyperactivity of platelets and, their adhesion and aggregation at the site of injury in atherosclerotic vessel walls, is critically important to the pathogenesis of $C V D[42,45,47,50,51]$. Furthermore, there is increasing evidence that acute clinical manifestations of coronary atherosclerotic disease are caused by plaque disruption and subsequent platelet-thrombus formation[52,53]. Coronary atherosclerotic lesions appear in early life and towards the end of the second decade of life; asymptomatic atherosclerosis lesions are present in most people living in industrialised societies. Platelet activity is thought to play a major role in the development as well as, in the stability of atherosclerotic plaques[52,53]. In support of the pathophysiological role of platelets, platelet inhibitory drugs such as aspirin have been observed to reduce the incidence of myocardial infraction, stroke and death from cardiovascular disease in secondary prevention trials[54]. However, the anti-platelet effects of aspirin are actually relatively weak and, the drug also has been shown to cause severe gastrointestinal disturbances and bleeding problems in some patients. Given the high incidence of cardiovascular disease in developed countries, there is great need for identification of effective antiplatelet compounds. These should inhibit platelet aggregation without increasing bleeding time (a problem associated with glycoprotein $\mathrm{Ilb} / \mathrm{Illa}$ antagonists) in order to circumvent problems with ulcers in patients who are taking aspirin as a prophylaxis[54]. Recently, aspirin's anti-platelet limitations have progressively underscored the critical need for improved platelet aggregation inhibitor therapy which is not only effective, but also safe and well tolerated. This concept has stimulated research into prevention of platelet hyperactivity by several means including dietary supplementation[55-59]. 
c. Effects of tomato extracts on human platelet aggregation

Table-2: Effects of fruit extracts on inhibition of human platelet aggregation in vitro \#\#.

\begin{tabular}{|c|c|c|c|}
\hline Fruit & Scientific names & $\%$ Fruit & $\begin{array}{c}\text { \% Inhibition of platelet } \\
\text { aggregation }\end{array}$ \\
\hline Tomato & Lycopersicon esculentum & 100 & $89.0 \pm 12.0$ \\
\hline Kiwifruit & Actinidia deliciosa & 100 & $75.0 \pm 5.0$ \\
\hline Grapefruit & Citrus paradisi & 100 & $44.2 \pm 8.1$ \\
\hline $\begin{array}{c}\text { Melon } \\
\text { (Honeydew) }\end{array}$ & Cucumis melo & 100 & $42.1 \pm 12.0$ \\
\hline Strawberry & Fragaria virginiana & 100 & $33.1 \pm 7.0$ \\
\hline $\begin{array}{c}\text { Melon } \\
\text { (Cantelope) }\end{array}$ & Cucumis melo cantalupensis & 100 & $27.5 \pm 11.0$ \\
\hline Banana & Musa paradisiaca & 50 & $22.4+5.0$ \\
\hline Mango & Mangifera indica & 50 & $22.1 \pm 6.3$ \\
\hline Pineapple & Ananas comosus & 100 & $19.8 \pm 9.4$ \\
\hline Orange (Jaffa) & Citrus sinensis cv. Jaffa & 100 & $18.5 \pm 7.6$ \\
\hline Grape (green) & Vitis vinifera & 100 & $16.4 \pm 8.9$ \\
\hline Plum & Prunus mexicana & 100 & $15.6 \pm 9.1$ \\
\hline Grape (red) & Vitis vinifera & 100 & $13.8 \pm 7.8$ \\
\hline Avocado & Persea americana & 20 & $12.1 \pm 4.5$ \\
\hline Nectarine & Prunus persica nucipersica & 50 & $9.1 \pm 4.2$ \\
\hline Apple & Malus domestica & 50 & $5.2 \pm 2.1$ \\
\hline Pear & Pyrus fauriei & 100 & $2.0 \pm 2.0$ \\
\hline
\end{tabular}

\#\# Adapted from [56,58,59] 
Table- 2 shows the anti-platelet aggregation effects of different fruit extracts on human platelets in vitro. The maximum inhibitory effect (70-75\%) was found to be with tomato and kiwi fruit extracts whereas, apple and pear had very little activity (2-5\%). Grapefruit, melon and strawberry had intermediate activities on platelet aggregation (33-44\%). The $\mathrm{pH}$ of the different fruit extracts was acidic in nature; therefore the $\mathrm{pH}$ of the extracts was adjusted to $\mathrm{pH} 7.4$ in order to avoid any effect of acidic $\mathrm{pH}$ on the platelet aggregation response. With the exception of avocado, apple, nectarine, banana and mango, a $100 \%$ juice $(\mathrm{w} / \mathrm{v})$ extract was used[8]. A dose-dependant inhibition of platelet aggregation was observed for the tomato extract; maximum inhibition (72\%) was obtained when 50 $\mu \mathrm{l}(100 \%$ juice) of tomato extract was added to $500 \mu \mathrm{l}$ of prepared platelets. Most of the anti-platelet properties of tomatoes reside in the juicy part of the fruit. The anti-platelet potential of the fruits tested appeared to be opposite to that of their antioxidant activity. According to the these authors, strawberry had the highest antioxidant capacity followed by plum, orange, red grape, kiwi fruit, pink grapefruit, white grape, banana, apple, tomato, pear, and honeydew melon. Since the anti-platelet activity in fruits is quite different from their antioxidant properties, it is possible that these activities are due to the presence of compounds that have a different chemical structure. Tomato extract used in a dose-dependant manner inhibited ADP-induced platelet aggregation in vitro. However, tomatoderived compounds also inhibit thrombin-induced platelet aggregation. Anti-thrombotic properties of tomato have also been demonstrated in a rat model. Delipidation of the tomato ultrafiltrate $(100,000 \times \mathrm{g})$ obtained from the tomato extract demonstrated that the anti-platelet factor(s) were not lipid-soluble compounds. The delipidated aqueous fraction was further purified by gel filtration using a Biogel P2 column. These anti-platelet compounds in tomato, had a molecular mass less than 1000da, were highly water soluble and were stable after boiling[8].

\section{d. Further characterisation of tomato extract}

The aqueous tomato extract consisted largely of soluble sugars (85-90\% of dry matter), which showed no in vitro anti-platelet activity[60]. The non-sugar material that was isolated (total active fraction, tAF) accounted for $4 \%$ of the aqueous extract dry matter and showed strong inhibition of platelet aggregation in vitro [60]. Most of the isolated components with antiplatelet activity are not flavonoid derivatives, and their characterization and individual antiplatelet activity profiles remain to be reported. A possible mechanism by which tomato extract components inhibit different pathways of platelet aggregation has been suggested from the observation that $\mathrm{TAF}$ and its subfractions prevent activation of integrin ⿴囗玉 $1 \mathrm{~b}_{3} \mathrm{~B}_{3}$ (ie, GPIIb/IIla). Expression of this integrin is specific to platelets and megakaryocytes. Its activation is essential for platelet aggregation, which it mediates by binding fibrinogen or von Willebrand factor (vWF). In resting platelets, GPIlb/IIla is maintained in a low- 
affinity state for ligand binding, which allows platelets to circulate freely. On activation, receptorspecific inside-out signals are generated, which activates GPIIb/IIla and increases its affinity for adhesive ligands. Inhibition of the GPIIb/IIla activation step-which is common to multiple aggregation pathways - could underlie the wide-ranging effects of tAF. The mechanism of action of these anti-platelet factors is not known, basal platelet cyclic AMP concentrations are unaltered by tomato extract active components in vitro, which suggests that phospholipase $C$ enzyme familymediated cascade reactions (which can affect GPIIb/IIla activation) may be at the root of the observed anti-aggregatory activity[61]. In addition to preventing platelet activation and aggregation, tAF reduces the expression of P-selectin (CD62P) on the platelet surface in response to ADP-induced platelet activation in whole blood. In resting platelets, P-selectin is localized in the membranes of platelet $\mathrm{Q}$-granules. On platelet activation, it is redistributed to the platelet surface, where it initiates adhesion to leukocytes. Under conditions of blood flow and shear stress, this glycoprotein promotes platelet cohesion and stabilizes newly formed aggregates. Thus, tAF components can potentially affect the size and longevity of platelet aggregates. The observed effects on P-selectin also imply that tAF components inhibit the release of alpha granule components in activated platelets, which affects many pro-aggregatory molecules.

\section{e. Human trials using tomato extract (Fruitflow)}

The ability of Fruitflow or tAF to modify the platelet response to stimulation by different agonists was evaluated by examining the changes in platelet aggregation response and expression of platelet activation markers. In addition, the ex vivo efficacy of the tomato extract was evaluated in a study designed to examine the acute effect of supplementation with tomato extract, which was given in an orange juice matrix. In a randomized, double-blinded, placebo-controlled crossover a significant reduction in platelet aggregation was observed $3 \mathrm{~h}$ after supplementation with tomato extract[60,62]. Two different extract-supplemented treatment drinks were prepared in 50- and 200$\mathrm{mL}$ volumes. Each treatment drink contained $18 \mathrm{~g}$ tomato extract syrup, which is equivalent to the quantity of tAF found in 6 fresh tomatoes. Inhibition of aggregation was observed for both ADP- and collagen-mediated aggregation in a dose-dependant manner. 


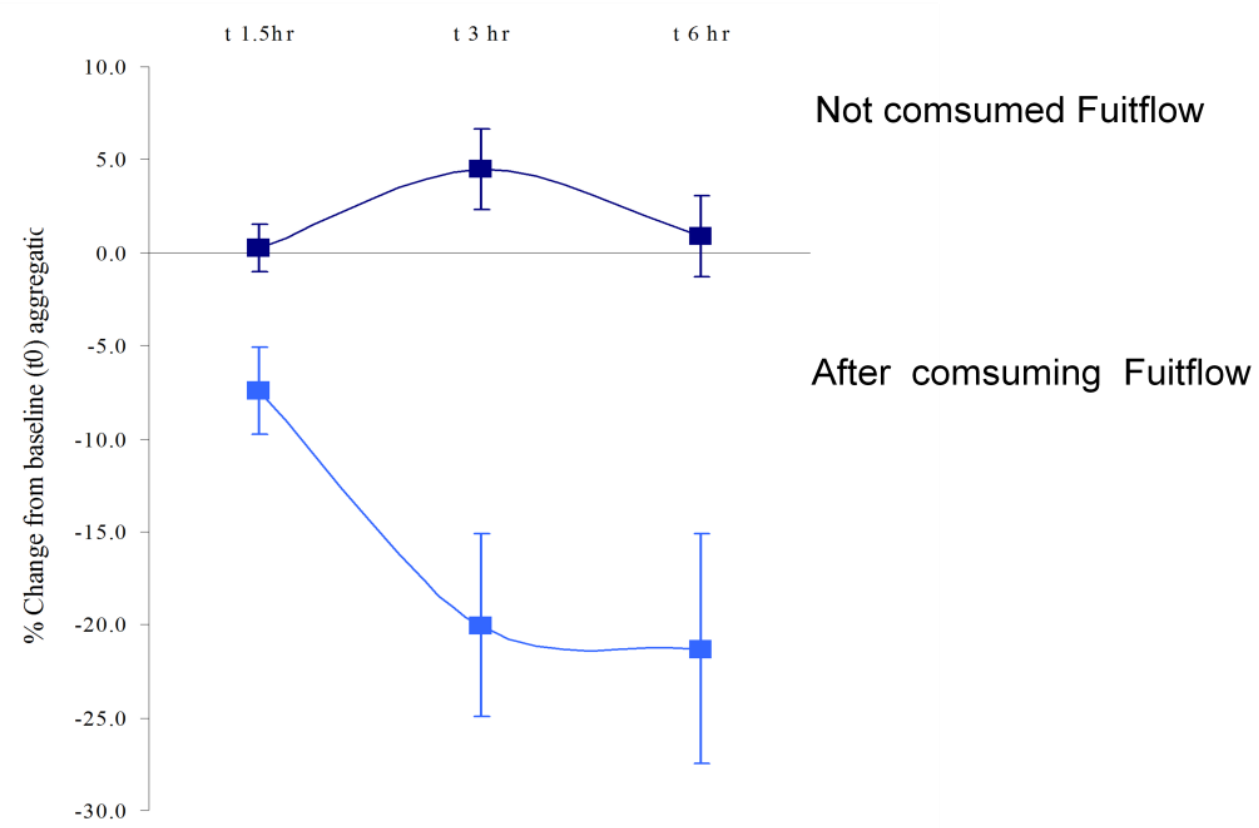

Figure. 2 shows the effect of tomato extract on human platelet aggregation after consumption. No adverse side-effects of the supplementation were reported, and no effects on clotting time variables were detected after supplementation.

\section{CONCLUSIONS}

Tomato contains several antioxidants including lycopene, flavonoids, and trace elements, vitamins beneficial to the cardiovascular system. In addition, the presence of several anti-platelet factors in tomatoes makes it ideal cardiovascular food. Modulation of platelet reactivity towards collagen, ADP and thrombin by tomato or tomato products is potentially prophylactic and therapeutic benefit in preventing and halting pathologic processes that lead to CVD. The human study showed that consumption of anti-platelet components derived from the tomato, in a supplement drink format suitable for use as a dietary supplement or functional food, led to a significant reduction in ex vivo platelet aggregation after $3 \mathrm{~h}$. The observed acute effects were more wide-ranging than those of aspirin, the only drug widely studied as a potential prophylactic, in that more than one pathway of platelet aggregation is targeted. Persons with high concentrations of some known markers of CVD showed greater sensitivity to supplementation, and overall the range of responses measured seemed appropriate for a primary prevention regime. Consumption of such tomato extracts as a food supplement could benefit public health by helping to maintain platelets in an inactivated state and reducing the risk of thrombotic events mediated by platelet activation. Hyperactivity of platelets is observed in people with diabetes, CVD, obesity, insulin resistance, smokers, and sedentary life styles. Therefore consumption of tomatoes will be beneficial to these people in preventing CVD. Recently European Food Safety Authority approved the claim that Fruitflow (tomato extract) helps to maintain a healthy blood flow and benefits circulation. Fruitflow is an absolutely unique dietary 
solution for cardiovascular support that works immediately after ingestion. Fruitflow works by helping to keep blood platelets smooth, thereby promoting healthy blood circulation in the body.

\section{REFERENCES}

[1] Canene-Adams K, Campbell JK, Zaripheh S, Jeffery EH, Erdman JW, Jr. The tomato as a functional food. Journal of Nutrition 2005; 135(5): 1226-30.

[2] Agarwal S, Rao AV. Tomato lycopene and low density lipoprotein oxidation: a human dietary intervention study. Lipids 1998; 33(10): 981-4.

[3] Mancini M, Parfitt VJ, Rubba P. Antioxidants in the Mediterranean diet. Canadain Journal of Cardiology1995; 11 Suppl G: 105G-9G.

[4] Verhoeyen ME, Bovy A, Collins G, Muir S, Robinson S, de Vos CH, Colliver S. Increasing antioxidant levels in tomatoes through modification of the flavonoid biosynthetic pathway. Journal of Experimental Botany 2002; 53(377): 2099-106.

[5] Blum A, Monir M, Wirsansky I, Ben-Arzi S. The beneficial effects of tomatoes. European Journal of Internal Medicine 2005; 16(6): 402-4.

[6] Agarwal S, Rao AV. Tomato lycopene and its role in human health and chronic diseases. Canadian Medical Association Journal 2000; 163(6): 739-44.

[7] Rissanen T, Voutilainen S, Nyyssonen K, Salonen JT. Lycopene, atherosclerosis, and coronary heart disease. Experimental Biology Medicine (Maywood) 2002; 227(10): 900-7.

[8] Duttaroy AK, Crosbie L, Gordon MJ. Effects of tomato extract on human platelet aggregation in vitro. Platelets $2001 ; 12(4): 218-27$.

[9] Giovannucci E. Tomatoes, tomato-based products, lycopene, and cancer: review of the epidemiologic literature. Journal of National Cancer Institute 1999; 91(4): 317-31.

[10] Weisburger JH. Lycopene and tomato products in health promotion. Experimental Biology and Medicine (Maywood 2002; 227(10): 924-7.

[11] Willcox JK, Catignani GL, Lazarus S. Tomatoes and cardiovascular health. Critical Reviews in Food science and Nutrition2003; 43(1): 1-18.

[12] Giovannucci E, Ascherio A, Rimm EB, Stampfer MJ, Colditz GA, Willett WC. Intake of carotenoids and retinol in relation to risk of prostate cancer. ournal of National Cancer Institute 1995; 87(23): 1767-76.

[13] Giovannucci E. A review of epidemiologic studies of tomatoes, lycopene, and prostate cancer. Experimental Biology Medicine (Maywood ) 2002; 227(10): 852-9.

[14] Cockey CD. Lycopene may contribute to heart health. Association of Women health Obstetric and Neonatal nurses Lifelines 2002; 6(3): 206. 
[15] La VC. Mediterranean epidemiological evidence on tomatoes and the prevention of digestive-tract cancers. Proceedings of the Society for Experimental Biology and Medicine 1998; 218(2): 125-8.

[16] Etminan M, Takkouche B, Caamano-Isorna F. The role of tomato products and lycopene in the prevention of prostate cancer: a meta-analysis of observational studies. Cancer Epidemiology Biomarkers and Prevention 2004; 13(3): 340-5.

[17] Rissanen TH, Voutilainen S, Nyyssonen K, Salonen R, Kaplan GA, Salonen JT. Serum lycopene concentrations and carotid atherosclerosis: the Kuopio Ischaemic Heart Disease Risk Factor Study. American Journal of Clinical Medicine 2003; 77(1): 133-8.

[18] Rao AV. Lycopene, tomatoes, and the prevention of coronary heart disease. Experimental Biology and Medicine (Maywood) 2002; 227(10): 908-13.

[19] Olfer'ev AM, Il'ina MV, Berzak NV, Stetsenko AV, Olfer'ev MA, Chudakova IA et al. [Effect of lycopene on blood lipoproteids in women with type 2 diabetes mellitus in postmenopause]. Voprosy pitaniia 2004; 73(1): 19-23.

[20] Maruyama C, Imamura K, Oshima S, Suzukawa M, Egami S, Tonomoto M et al. Effects of tomato juice consumption on plasma and lipoprotein carotenoid concentrations and the susceptibility of low density lipoprotein to oxidative modification. Journal of Nutritional Science and Vitaminology (Tokyo) 2001; 47(3): 213-21.

[21] Giugliano D. Dietary antioxidants for cardiovascular prevention. Nutrition, Metabolism and Cardiovascular Diseases2000; 10(1): 38-44.

[22] Kardinaal AF, Kok FJ, Ringstad J, Gomez-Aracena J, Mazaev VP, Kohlmeier L et al. Antioxidants in adipose tissue and risk of myocardial infarction: the EURAMIC Study. Lancet 1993; 342(8884): 1379-84.

[23] Kardinaal AF, Aro A, Kark JD, Riemersma RA, van ', V, Gomez-Aracena J et al. Association between beta-carotene and acute myocardial infarction depends on polyunsaturated fatty acid status. The EURAMIC Study. European Study on Antioxidants, Myocardial Infarction, and Cancer of the Breast. Arteriosclerosis Thrombosis and Vascular Biology 1995; 15(6): 72632.

[24] Kohlmeier L, Kark JD, Gomez-Gracia E, Martin BC, Steck SE, Kardinaal AF et al. Lycopene and myocardial infarction risk in the EURAMIC Study. American Journal of Epidemiology1997; 146(8): 618-26.

[25] Kristenson M, Zieden B, Kucinskiene Z, Elinder LS, Bergdahl B, Elwing B et al. Antioxidant state and mortality from coronary heart disease in Lithuanian and Swedish men: concomitant cross sectional study of men aged 50. British Medical Journal 1997; 314(7081): 629-33.

[26] Schmidt R, Fazekas F, Hayn M, Schmidt H, Kapeller P, Roob G et al. Risk factors for microangiopathy-related cerebral damage in the Austrian stroke prevention study. Journal of Neurological Sciences 1997; 152(1): 15-21. 
[27] Heber D, Lu QY. Overview of mechanisms of action of lycopene. Experimental Biology and Medicine (Maywood) 2002; 227(10): 920-3.

[28] Arab L, Steck S. Lycopene and cardiovascular disease. American Journal of Clinical Nutrition 2000; 71(6 Suppl): 1691S-5S.

[29] King DE. Dietary fiber, inflammation, and cardiovascular disease. Molecular Nutrition and Food Research2005; 49(6): 594-600.

[30] Pearson TA, Osorio D, Brown K. Nutritional interventions in cardiovascular disease: new challenges and opportunities. Current Atherosclerosis Reports 2000; 2(6): 515-20.

[31] Roche HM, Phillips C, Gibney MJ. The metabolic syndrome: the crossroads of diet and genetics. The Proceedings of the Nutrition Society 2005; 64(3): 371-7.

[32] Jialal I, Fuller CJ. Oxidized LDL and antioxidants. Clin Cardiol 1993; 16(4 Suppl 1): I6-I9.

[33] Molavi B, Mehta JL. Oxidative stress in cardiovascular disease: molecular basis of its deleterious effects, its detection, and therapeutic considerations. Current Opinion in Cardiology 2004; 19(5): 488-93.

[34] Nuttall SL, Kendall MJ, Martin U. Antioxidant therapy for the prevention of cardiovascular disease. QJM 1999; 92(5): 239-44.

[35] Shen YC, Chen SL, Wang CK. Contribution of tomato phenolics to antioxidation and downregulation of blood lipids. Journal of Agriculture Food Chemistry 2007; 55(16): 6475-81.

[36] Duttaroy AK, Kahn NN, Sinha AK. Prostaglandin-E1 - the Endogenous Physiological Regulator of Platelet Mediated Blood-Coagulation. Prostaglandins Leukotrienes and Essential Fatty Acids 1989; 35(4): 189-95.

[37] Hamet P, Sugimoto H, Umeda F, Franks DJ. Platelets and vascular smooth muscle: abnormalities of phosphodiesterase, aggregation, and cell growth in experimental and human diabetes. Metabolism 1983; 32(7 Suppl 1): 124-30.

[38] Kroll MH, Schafer Al. Biochemical mechanisms of platelet activation. Blood 1989; 74(4): 1181-95.

[39] Coller BS, Anderson K, Weisman HF. New antiplatelet agents: platelet GPIIb/IIla antagonists. Thrombosis and Haemostosis 1995; 74(1): 302-8.

[40] Duttaroy AK, Ray TK, Sinha AK. Prostacyclin Stimulation of the Activation of BloodCoagulation Factor-X by Platelets. Science 1986; 231(4736): 385-8.

[41] Duttaroy AK, Sinha AK. Purification and Properties of Prostaglandin-E1 Prostacyclin Receptor of Human-Blood Platelets. Journal of Biological Chemistry 1987; 262(26): 12685-91.

[42] Duttaroy AK. Insulin-Mediated Processes in Platelets, Erythrocytes and Monocytes Macrophages - Effects of Essential Fatty-Acid Metabolism. Prostaglandins Leukotrienes and Essential Fatty Acids 1994; 51(6): 385-99. 
[43] Duttaroy AK, Gordon MJ, Campbell FM, Crosbie LC. Arachidonic acid uptake by human platelets is mediated by CD36. Platelets 1996; 7(5-6): 291-5.

[44] Harker LA, Fuster V. Pharmacology of platelet inhibitors. Journal of American College Cardioliology 1986; 8(6 Suppl B): 21B-32B.

[45] Duttaroy AK, Kahn NN, Sinha AK. Interaction of Receptors for Prostaglandin-E1 Prostacyclin and Insulin in Human Erythrocytes and Platelets. Life Sciences 1991; 49(16): 1129-39.

[46] Hamberg M, Svensson J, Samuelsson B. Letter: Mechanism of the anti-aggregating effect of aspirin on human platelets. Lancet 1974; 2(7874): 223-4.

[47] Badimon JJ, Zaman A, Helft G, Fayad Z, Fuster V. Acute coronary syndromes: pathophysiology and preventive priorities. Thrombosis and Haemostosis 1999; 82(2): 9971004.

[48] Falk E, Shah PK, Fuster V. Coronary plaque disruption. Circulation 1995; 92(3): 657-71.

[49] Schror K. Aspirin and platelets: the antiplatelet action of aspirin and its role in thrombosis treatment and prophylaxis. Seminar in Thrombosis and Hemostasis 1997; 23(4): 349-56.

[50] Pierre S, Crosbie L, Duttaroy AK. Inhibitory effect of aqueous extracts of some herbs on human platelet aggregation in vitro. Platelets 2005; 16(8): 469-73.

[51] Duttaroy AK, Jorgensen A. Effects of kiwi fruit consumption on platelet aggregation and plasma lipids in healthy human volunteers. Platelets 2004; 15(5): 287-92.

[52] Duttaroy AK, Gordon MJ, Kelly C, Hunter K, Crosbie L, Knight-Carpentar T, Williams BC. Inhibitory effect of Ginkgo biloba extract on human platelet aggregation. Platelets 1999; 10(5): 298-305.

[53] Duttaroy AK, Crosbie L, Gordon MJ. Effects of tomato extract on human platelet aggregation in vitro. Platelets 2001; 12(4): 218-27.

[54] Duttaroy AK. Dietary components and human platelet activity. Platelets 2002; 13(2): 67-75.

[55] O'Kennedy N, Crosbie L, van LM, Broom JI, Webb DJ, Duttaroy AK. Effects of antiplatelet components of tomato extract on platelet function in vitro and ex vivo: a time-course cannulation study in healthy humans. American Journal of Clinical Nutrition 2006; 84(3): 570-9.

[56] Lazarus SA, Garg ML. Tomato extract inhibits human platelet aggregation in vitro without increasing basal cAMP levels. International Journal of Food Science and Nutrition 2004; 55(3): 249-56.

[57] O'Kennedy N, Crosbie L, Whelan S, Luther V, Horgan G, Broom Jl et al. Effects of tomato extract on platelet function: a double-blinded crossover study in healthy humans. American Journal of Clinical Nutrition 2006; 84(3): 561-9. 